\title{
Optimal Constants in Khintchine Type Inequalities for Fermions, Rademachers and $q$-Gaussian Operators
}

\section{Artur BUCHHOLZ}

\author{
Presented by Stanisław KWAPIEN
}

Summary. For $\left(P_{k}\right)$ being Rademacher, Fermion or $q$-Gaussian $(-1 \leq q \leq 0)$ operators, we find the optimal constants $C_{2 n}, n \in \mathbb{N}$, in the inequality

$$
\left\|\sum_{k=1}^{N} A_{k} \otimes P_{k}\right\|_{2 n} \leq\left[C_{2 n}\right]^{1 / 2 n} \max \left\{\left\|\left(\sum_{k=1}^{N} A_{k}^{*} A_{k}\right)^{1 / 2}\right\|_{L_{2 n}},\left\|\left(\sum_{k=1}^{N} A_{k} A_{k}^{*}\right)^{1 / 2}\right\|_{L_{2 n}}\right\},
$$

valid for all finite sequences of operators $\left(A_{k}\right)$ in the non-commutative $L_{2 n}$ space related to a semifinite von Neumann algebra with trace. In particular, $C_{2 n}=(2 n-1)$ !! for the Rademacher and Fermion sequences.

Introduction. The classical Khintchine inequality states that

$$
\left\|\sum \alpha_{k} r_{k}\right\|_{L_{p}(0,1)} \leq\left(2\left\lceil\frac{p}{2}\right\rceil-1\right) ! !\left(\sum \alpha_{k}^{2}\right)^{1 / 2}
$$

for $p \geq 2$ and all finite sequences $\left(\alpha_{k}\right)$ of real scalars. $\left(r_{k}\right)$ is the Rademacher sequence, i.e.

$$
r_{k}(x)=\operatorname{sgn}\left(\sin \left(2^{k} \pi x\right)\right), \quad k=1,2, \ldots
$$

The inequality appears in many branches of mathematical analysis and it was generalized in many different ways. Also a lot of effort was put into improving the constants in these inequalities.

The first such generalization is due to Orlicz who replaced the sequence of scalars $\left(\alpha_{k}\right)$ by a sequence of vectors in the Banach space $L_{p}$.

2000 Mathematics Subject Classification: Primary 46L53; Secondary 46L52.

Key words and phrases: Khintchine inequality, non-commutative analysis, commutation relations, Rademacher functions.

Research partially supported by KBN grant 2P03A00723 and HPRN-CT-2002-00279. 
A natural generalization of the Orlicz inequality is the result of F. LustPiquard [LP] who proved

$$
\left\|\sum_{k=1}^{N} A_{k} \otimes r_{k}\right\|_{p} \leq C_{p} \max \left\{\left\|\left(\sum_{k=1}^{N} A_{k}^{*} A_{k}\right)^{1 / 2}\right\|_{L_{p}},\left\|\left(\sum_{k=1}^{N} A_{k} A_{k}^{*}\right)^{1 / 2}\right\|_{L_{p}}\right\},
$$

where $A_{k}$ are operators from the Schatten trace class $L_{p}$. However the constants $C_{p}$ she obtained are quite large.

In this paper we give the proof of this inequality for $p=2 n$ which gives $C_{2 n}=(2 n-1)$ !!. For this we show that in the inequality (1) one can replace the Rademacher sequence by the Fermi sequence $\left(U_{k}\right)$ (see formulas $(3)$ for a definition). This follows from the fact that the Rademacher sequence has the same distribution as the sequence $\left(U_{k} \otimes U_{k}\right)$ (see Lemma 3 and Corollary 4 ).

In the last section we study Khintchine type inequalities for $q$-Gaussian sequences with negative $q$. We find the optimal constants in this case.

Definitions and notations. Let $H_{\mathbb{R}}$ be a real Hilbert space with $\operatorname{dim} H_{\mathbb{R}}=N$, and let $H$ be its complexification. The inner product on $H$ will be denoted by $\langle\cdot \mid \cdot\rangle_{H}$. We define the vector space $\mathcal{F}^{0}$ by

$$
\mathcal{F}^{0}=\mathbb{C} \Omega \oplus \bigoplus_{k=1}^{\infty} H^{\otimes k}
$$

where $\mathbb{C} \Omega=H^{\otimes 0}$ is the 1 -dimensional space spanned by a fixed vector $\Omega$. We choose an orthonormal basis $\left\{e_{1}, \ldots, e_{N}\right\}$ for $H_{\mathbb{R}}$, and we define the linear operators $l_{k}^{q}, l_{k}^{+}(k \in\{1, \ldots, N\}, q \in[-1,1])$ acting on $\mathcal{F}^{0}$ by the formulas

$$
\begin{aligned}
l_{k}^{+} \Omega & =e_{k}, \\
l_{k}^{+} e_{i_{1}} \otimes \cdots \otimes e_{i_{m}} & =e_{k} \otimes e_{i_{1}} \otimes \cdots \otimes e_{i_{m}}, \\
l_{k}^{q} \Omega & =0 \\
l_{k}^{q} e_{m} & =\left\langle e_{m} \mid e_{k}\right\rangle_{H} \Omega, \\
l_{k}^{q} e_{i_{1}} \otimes \cdots \otimes e_{i_{m}} & =\sum_{p=1}^{m} q^{p-1}\left\langle e_{i_{p}} \mid e_{k}\right\rangle_{H} e_{i_{1}} \otimes \cdots \otimes e_{i_{p-1}} \otimes \hat{e}_{i_{p}} \otimes e_{i_{p+1}} \otimes e_{i_{m}},
\end{aligned}
$$

where the hat indicates omission of a vector in the tensor product. Next, we introduce a non-negative Hermitian form $\langle\cdot \mid \cdot\rangle_{\mathcal{F}_{q}}$ on $\mathcal{F}^{0}$, and we define a Hilbert space $\mathcal{F}_{q}$. Namely, whenever $k \neq l$, the subspaces $H^{\otimes k}$ and $H^{\otimes l}$ are orthogonal with respect to $\langle\cdot \mid \cdot\rangle_{\mathcal{F}_{q}}$, the vector $\Omega$ has norm 1 with respect to this form, and on $H^{\otimes k}$ we put

$$
\left\langle e_{i_{1}} \otimes \cdots \otimes e_{i_{k}} \mid e_{j_{1}} \otimes \cdots \otimes e_{j_{k}}\right\rangle_{\mathcal{F}_{q}}=\sum_{\sigma \in S_{k}} q^{i(\sigma)} \prod_{p=1}^{k}\left\langle e_{i_{p}} \mid e_{j_{\sigma(p)}}\right\rangle_{H},
$$


where $S_{k}$ is the symmetric group, and $i(\sigma)$ is the number of inversions in the permutation $\sigma$, i.e.,

$$
i(\sigma)=\#\{(k, l): k<l \text { and } \sigma(k)>\sigma(l)\} .
$$

This form has non-trivial kernel when $q \in\{-1,1\}$. Note also that $H^{\otimes k}=0$ in the Hilbert space $\mathcal{F}_{-1}$ whenever $k>N$. The operators $l_{k}^{q}$ and $l_{k}^{+}$preserve the kernel of the form $\langle\cdot \mid \cdot\rangle_{\mathcal{F}_{q}}$. This allows us to consider $l_{k}^{q}$ and $l_{k}^{+}$as operators on the space $\mathcal{F}_{q}$, defined to be the completion of $\mathcal{F}^{0} / \operatorname{ker}\langle\cdot \mid \cdot\rangle_{\mathcal{F}_{q}}$ in the norm given by the scalar product $\langle\cdot \mid \cdot\rangle_{\mathcal{F}_{q}}$. From now on $l_{k}^{q}$ and $l_{k}^{+}$stand for these operators on $\mathcal{F}_{q}$. They are mutually adjoint,

$$
\left(l_{k}^{q}\right)^{*}=l_{k}^{+}
$$

The case $q=1$ is special since our operators are unbounded.

DEFINITION 1. Let $\left\{e_{1}, \ldots, e_{N}\right\}$ be an orthonormal basis of the real Hilbert space $H_{\mathbb{R}}$, and let $H$ be the complexification of $H_{\mathbb{R}}$. The sequence $\left(G_{k}^{q}\right)_{k=1}^{N}$ of selfadjoint operators defined by

$$
G_{k}^{q}=l_{k}^{q}+l_{k}^{+}
$$

where $l_{k}^{q}$ and $l_{k}^{+}$are as above, is called the $q$-Gaussian operator sequence. In the case $q=-1$ those operators will be called fermions and denoted by $U_{k}$.

The following formula for mixed moments of $q$-Gaussian operators will be used (see [BSp1]):

$$
\left\langle G_{k_{1}}^{(q)} \cdots G_{k_{2 m}}^{(q)} \Omega \mid \Omega\right\rangle=\sum_{\substack{\nu \in \mathbb{P}_{2}(2 m) \\ \nu=\left\{\left\{p_{1}, q_{1}\right\}, \ldots\left\{p_{m}, q_{m}\right\}\right\}}} q^{i(\nu)} \prod_{s=1}^{m}\left\langle e_{k_{p_{s}}} \mid e_{k_{q_{s}}}\right\rangle_{H},
$$

where $\mathbb{P}_{2}(2 m)$ is the set of 2 -partitions of the set $\{1, \ldots, 2 m\}$, and $i(\nu)$ is the crossing number of the 2-partition $\nu$.

In $[\mathrm{Bu}]$ a Khintchine type inequality for $q$-Gaussian operator sequences was proved. In the last section we will show the optimality for $q<0$ in the following theorem (see Theorem 5 of $[\mathrm{Bu}]$ ):

THEOREM 2. Let $\left(G_{k}^{q}\right)_{k=1}^{N}$ be the $q$-Gaussian operator sequence, and let $\left(A_{k}\right)_{k=1}^{N}$ be a sequence of operators from the non-commutative $L_{2 n}$-space related to a semifinite von Neumann algebra with trace Tr. Then

$$
\begin{aligned}
& \left\|\sum_{k=1}^{N} A_{k} \otimes G_{k}^{q}\right\|_{2 n} \\
& \quad \leq\left[C_{2 n}(q)\right]^{1 / 2 n} \max \left\{\left\|\left(\sum_{k=1}^{N} A_{k}^{*} A_{k}\right)^{1 / 2}\right\|_{L_{2 n}},\left\|\left(\sum_{k=1}^{N} A_{k} A_{k}^{*}\right)^{1 / 2}\right\|_{L_{2 n}}\right\},
\end{aligned}
$$


where $\|T\|_{2 n}^{2 n}=\operatorname{Tr} \otimes \mathbb{E}_{\Omega}\left(\left(T T^{*}\right)^{n}\right)$ for the state $\mathbb{E}_{\Omega}(S)=\langle S \Omega \mid \Omega\rangle_{\mathcal{F}_{q}}$ and

$$
C_{2 n}(q)=\left\langle\left(G_{1}^{|q|}\right)^{2 n} \Omega \mid \Omega\right\rangle .
$$

Moreover the constant $C_{2 n}(q)$ in the above inequality is optimal for nonnegative $q$.

Fermions. In this section we consider the case $q=-1$. We will write $U_{k}$ instead of $G_{k}^{-1}$. The main properties of the operators $U_{k}$ are their unitarity and commutation properties, i.e.

$$
\begin{aligned}
U_{k} & =U_{k}^{*}, \\
U_{k} U_{k} & =\mathbb{1}, \\
U_{k} U_{l} & =-U_{l} U_{k}, \quad k \neq l,
\end{aligned}
$$

where $\mathbb{1}$ is the identity operator on $\mathcal{F}_{-1}$. The next lemma establishes a relationship between the Fermion sequence and the Rademacher sequence.

Lemma 3. Let $\left(U_{k}\right)_{k=1}^{N}$ and $\left(r_{k}\right)_{k=1}^{N}$ be the Fermion and Rademacher sequences respectively. Moreover let $G_{R}$ be the group generated by the operators $R_{k}=U_{k} \otimes U_{k}$ acting on the Hilbert space $\mathcal{F}_{-1} \otimes_{2} \mathcal{F}_{-1}$, and let $G_{r}$ be the group under pointwise multiplication generated by the rademachers $r_{k}$. Then the mapping

$$
R_{k} \mapsto r_{k}
$$

extends to a group isomorphism between $G_{R}$ and $G_{r}$.

Proof. By (3), $R_{k}$ have order two and form a commuting family. We have to check that for any $m \leq N$,

$$
R_{k_{1}} \cdots R_{k_{m}} \neq \mathbb{1}
$$

where $k_{1}, \ldots, k_{m}$ are different positive integers. This follows from

$$
R_{k_{1}} \cdots R_{k_{m}}[\Omega] \otimes[\Omega]=\left[e_{k_{1}} \otimes \cdots \otimes e_{k_{m}}\right] \otimes\left[e_{k_{1}} \otimes \cdots \otimes e_{k_{m}}\right] \neq[\Omega] \otimes[\Omega] .
$$

The above lemma can be equivalently stated as follows:

COROLlary 4. The joint distribution of the Rademacher sequence is the same as the distribution of the sequence $\left(R_{k}\right)$, i.e.

$$
\left\langle R_{k_{1}} \cdots R_{k_{m}}[\Omega] \otimes[\Omega] \mid[\Omega] \otimes[\Omega]\right\rangle_{\mathcal{F} \otimes \mathcal{F}}=\int_{0}^{1} r_{k_{1}}(t) \cdots r_{k_{m}}(t) d t .
$$

From now on, the symbol $C_{2 n}$ will denote the number $(2 n-1)$ !!. Theorem 2 and Corollary 4 imply 
THEOREM 5. Let $\left(r_{k}\right)_{k=1}^{N}$ be the Rademacher sequence. Moreover let $\left(A_{k}\right)_{k=1}^{N}$ be as in the preceding theorem. Then

$$
\begin{aligned}
& \left\|\sum_{k=1}^{N} A_{k} \otimes r_{k}\right\|_{2 n} \\
& \quad \leq\left(C_{2 n}\right)^{1 / 2 n} \max \left\{\left\|\left(\sum_{k=1}^{N} A_{k}^{*} A_{k}\right)^{1 / 2}\right\|_{L_{2 n}},\left\|\left(\sum_{k=1}^{N} A_{k} A_{k}^{*}\right)^{1 / 2}\right\|_{L_{2 n}}\right\} .
\end{aligned}
$$

Moreover the constant $C_{2 n}=(2 n-1)$ !! is optimal as $N$ runs over the natural numbers and $\left(A_{k}\right)$ runs over the non-commutative $L_{2 n}$-spaces.

Proof. By Corollary 4 we have

$$
\begin{aligned}
\left\|\sum_{k=1}^{N} A_{k} \otimes r_{k}\right\|_{2 n}^{2 n} & =\operatorname{Tr} \int_{0}^{1}\left(\left(\sum_{k=1}^{N} A_{k} r_{k}(t)\right)\left(\sum_{k=1}^{N} A_{k}^{*} r_{k}(t)\right)\right)^{n} d t \\
& =\left\|\sum_{k=1}^{N} A_{k} \otimes U_{k} \otimes U_{k}\right\|_{2 n}^{2 n} \cdot
\end{aligned}
$$

The equality above, Theorem 2 applied to $A_{k}$ replaced by $A_{k} \otimes U_{k}$, and the first equality in (3) complete the proof.

Also, as a consequence of the above proof we obtain equality between the optimal constants in the operator Khintchine inequality for fermions and rademachers as well as the optimality of the constants $C_{2 n}(-1)=(2 n-1)$ !! in Theorem 2.

TheOREM 6. For any $p \geq 2$ the optimal constants in the operator Khintchine inequality for the Fermion and Rademacher sequences are identical.

Proof. As was mentioned above, the equality (5) implies that for any even polynomial $w$ and any bounded sequence $\left(B_{k}\right)$,

$$
\operatorname{Tr} \otimes \int\left(w\left(\sum B_{k} \otimes r_{k}\right)\right)=\operatorname{Tr} \otimes \mathbb{E}_{\Omega} \otimes \mathbb{E}_{\Omega}\left(w\left(\sum B_{k} \otimes U_{k} \otimes U_{k}\right)\right) .
$$

Since the function $|\cdot|^{p}$ can be uniformly approximated on compact sets by even polynomials and since $L_{\infty}$ is dense in $L_{p}$ we get the assertion.

REMARK 7. The constant $C_{2 n}(-1)$ is optimal in Theorem 2.

q-Gaussian. We will make use of the following non-commutative Central Limit Theorem (see Theorem 0 in [BSp1]).

THEOREM 8. Let $\mathcal{B}$ be a unital *-algebra with a state $\phi$. Consider selfadjoint elements $b_{i}=b_{i}^{*} \in \mathcal{B} i \in \mathbb{N}$ normalized by $\phi\left(b_{i}^{2}\right)=1$, which satisfy the following assumptions: 


$$
\begin{aligned}
& \phi\left(b_{i_{1}} \cdots b_{i_{n}}\right)=0 \quad \text { whenever } \quad \#\left\{m: i_{k}=i_{m}\right\}=1 \text { for some } k, \\
& \phi\left(b_{i_{1}} \cdots b_{i_{n}}\right)=\phi\left(b_{\pi\left(i_{1}\right)} \cdots b_{\pi\left(i_{n}\right)}\right) \quad \text { for any injection } \pi: \mathbb{N} \rightarrow \mathbb{N} .
\end{aligned}
$$

Then for the operators

$$
S_{N}(k)=\frac{1}{\sqrt{N}} \sum_{i \in A_{N, k}} b_{i}
$$

where for each $N$ the sets $A_{N, k}$ are disjoint and of cardinality $N$ each, the following equalities hold:

$$
\begin{gathered}
\lim _{N \rightarrow \infty} \phi\left(S_{N}\left(k_{1}\right) \cdots S_{N}\left(k_{n}\right)\right)=0 \quad \text { whenever } n \in 2 \mathbb{N}+1, \\
\lim _{N \rightarrow \infty} \phi\left(S_{N}\left(k_{1}\right) \cdots S_{N}\left(k_{2 n}\right)\right)=\sum_{\nu \in \mathbb{P}_{2}(\{1, \ldots, 2 n\})} \delta_{k_{i_{1}}, k_{j_{1}}} \cdots \delta_{k_{i_{n}}, k_{j_{n}}} t(\nu),
\end{gathered}
$$

where $\nu=\left\{\left\{i_{1}, j_{1}\right\}, \ldots,\left\{i_{n}, j_{n}\right\}\right\}$ and $t$ is some positive definite function on $\mathbb{P}_{2}$.

To show the optimality of the constants $C_{2 n}(q)=\left\langle\left(G_{1}^{|q|}\right)^{2 n} \Omega \mid \Omega\right\rangle^{1 / 2 n}$ in Theorem 2 we will follow the method used for fermions.

Consider the operators

$$
R_{k}^{q}=U_{k} \otimes G_{k}^{q}
$$

Since the sequence $\left(R_{k}^{q}\right)_{k=1}^{\infty}$ satisfies the assumptions of Theorem 8 , the optimal constants in Theorem 2 cannot be smaller than the moments of the central measure associated with the sequence $\left(R_{k}^{q}\right)_{k=1}^{\infty}$. By (3) and (2) this measure has the $2 n$-moments equal to the corresponding moments for the operator $G_{1}^{|q|}$,

$$
\left\langle\left(\lim _{N \rightarrow \infty} \frac{1}{\sqrt{N}} \sum_{k=1}^{N} R_{k}^{q}\right)^{2 n}[\Omega] \otimes \Omega \mid[\Omega] \otimes \Omega\right\rangle=\left\langle\left(G_{1}^{|q|}\right)^{2 n} \Omega \mid \Omega\right\rangle .
$$

The above considerations prove the following theorem:

THEOREM 9. The constants in Theorem 2 remain optimal when $q \in$ $[-1,1]$.

\section{References}

[B] M. Bożejko, On $\Lambda(p)$ sets with the minimal constant in discrete noncommutative groups, Proc. Amer. Math. Soc. 51 (1975), 407-412.

[BSp1] M. Bożejko and R. Speicher, Interpolations between bosonic and fermionic relations given by generalized Brownian motions, Math. Z. 222 (1996), 135-159.

[Bu] A. Buchholz, Operator Khintchine inequality in non-commutative probability, Math. Ann. 319 (2001), 1-16.

[LP] F. Lust-Piquard, Inégalités de Khintchine dans $C_{p}(1<p<\infty)$, C. R. Acad. Sci. Paris 303 (1986), 289-292. 
[P] G. Pisier, An inequality for p-orthogonal sums in non-commutative $L_{p}$, Illinois J. Math. 44 (2000), 901-923.

\author{
Artur Buchholz \\ Institute of Mathematics \\ University of Wrocław \\ Pl. Grunwaldzki 2/4 \\ 50-384 Wrocław, Poland \\ E-mail: buchholz@math.uni.wroc.pl
}

Received December 17, 2004;

received in final form September 8, 2005 